\title{
EFFECTIVENESS OF SELF-EXPANDING METALLIC STENTS IN THE PALLIATION OF ABSOLUTE DYSPHAGIA IN CARCINOMA OESOPHAGUS- A 1-YEAR FOLLOW-UP STUDY
}

\author{
Pauly Thazhungal Joseph ${ }^{1}$, Santhosh Thekkoot Vijayan'2, Sudhiraj T. S ${ }^{3}$
}

${ }_{1}^{1}$ Associate Professor, Department of Surgery, Government Medical College, Thrissur, Kerala.

${ }^{2}$ Associate Professor, Department of Surgery, Government Medical College, Thrissur, Kerala.

${ }^{3}$ Assistant Professor, Department of Community Medicine, Government Medical College, Thrissur, Kerala.

ABSTRACT

\section{BACKGROUND}

Palliation of dysphagia is the primary goal in the care of inoperable oesophageal carcinoma and the usual option is palliative chemoradiotherapy. But radiation induced complications made chemoradiation a less favourable option for managing dysphagia. Rapid improvement in dysphagia with minimal complications and technical simplicity made SEMS the primary option in the palliation of dysphagia.

The aim of this study was to assess the outcomes of inoperable oesophageal malignancy presented with absolute dysphagia, who were treated with SEMS placement. The objective of our study was to assess the effectiveness of SEMS in palliation of Grade 4 dysphagia due to oesophageal carcinoma. Other aims were the technical success rate, complications and survival.

\section{MATERIALS AND METHODS}

This is a single group cohort study done in the Department of General Surgery, Government Medical College, Thrissur, during a period of 1 year from 1st June 2013 to 31st May 2014, with a mean follow-up of 1 year. Patients presented to Surgery Department with absolute dysphagia due to carcinoma oesophagus, offered palliative SEMS. Clinical success rate, technical success rate, complications and survival was analysed.

\section{RESULTS}

34 patients were selected for stenting based on inclusion and exclusion criteria. We were able to place SEMS successfully in 29 patients. The technical success rate was $85.29 \%$. A clinical success rate was $96.55 \%$. Post stent score was Gd 3 in 19 (65.5\%), Gd 2 in 7 (24.1\%), Gd 1 in $2(6.9 \%)$ and Gd 4 in 1 (3.4\%). Wilcoxon Signed Rank Test ' $z$ ' value was 4.813 (p value $<0.001$ ). Median survival was 20 weeks (95\%, CI 12.08 - 27.91). There was one procedure related mortality due to oesophageal perforation. Incidence of recurrent dysphagia in our study was $17.24 \%$. It was due to stent migration in $2(06.89 \%)$ and tumour overgrowth in $3(10.34 \%)$.

\section{CONCLUSION}

SEMS is an effective method in palliation of dysphagia due to oesophageal carcinoma. Significance of this study is that it specifically examines the effectiveness of SEMS in a group of patients with absolute dysphagia as a primary palliative modality. This aspect is important considering the limited radiation facilities and high cost of chemotherapy in emerging nations like ours.

\section{KEYWORDS}

Carcinoma Oesophagus, Palliation Dysphagia, SEMS, Survival.

HOW TO CITE THIS ARTICLE: Joseph PT, Vijayan ST, Sudhiraj TS. Effectiveness of self-expanding metallic stents in the palliation of absolute dysphagia in carcinoma oesophagus- a 1-year follow-up study. J. Evolution Med. Dent. Sci. 2018;7(11):1400-1403, DOI: $10.14260 /$ jemds/2018/318

\section{BACKGROUND}

Carcinoma oesophagus is the eighth most common malignancy worldwide and accounts for sixth leading cause of deaths. In India it is the fourth most common cause for cancer related mortality with the annual incidence of 47,000 per year and 42,000 deaths annually. $(1,2)$

Dysphagia is the most common presenting symptom of more than $2 / 3$ of patients with oesophageal carcinoma resulting in malnutrition and loss of weight. Other symptoms are chest pain, aspiration and symptoms due to tracheooesophageal fistula. Early in the course of disease,

'Financial or Other Competing Interest': None.

Submission 17-01-2018, Peer Review 21-02-2018,

Acceptance 27-02-2018, Published 12-03-2018.

Corresponding Author:

Dr. Santhosh Thekkoot Vijayan,

Thekkoot CH, Kuzhikkattussery P. O.,

Thrissur-680697.

E-mail:drsan72@gmail.com

DOI: $10.14260 /$ jemds $/ 2018 / 318$ oesophageal carcinoma remains asymptomatic and evades diagnosis. By the time it exhibits symptoms, it will not be amenable to curative resection. At the time of the diagnosis more than $50 \%$ of patients will be in an inoperable stage, either due to locally advanced stage or metastasis.(3)

Palliation of dysphagia is the primary goal in the care of inoperable oesophageal carcinoma and the usual option is palliative chemoradiotherapy. But it will take longer time and repeated sessions to control the disease and relieve dysphagia. Along with this, radiation induced complications made chemoradiation a less favourable option for managing dysphagia.(4) Other non-surgical methods are Laser therapy, Photodynamic treatment, intralesional injection of alcohol and stenting. Availability, cost-effectiveness and minimal risk for complications are the considerations in selecting the treatment modalities and sometimes it may need more than one modality. Since the introduction of SEMS in the early 1990s, it became a well-accepted modality in the management of dysphagia due to malignant stricture. Rapid 
improvement in dysphagia with minimal complications and technical simplicity made SEMS the primary option in the palliation of dysphagia. ${ }^{(5)}$

\section{Aims and Objectives}

The aim of our study was to assess the effectiveness of SEMS in palliation of Grade 4 dysphagia due to oesophageal carcinoma. The objectives were the clinical success rate, the technical success rate, complications and survival.

\section{MATERIALS AND METHODS}

This is a single group cohort study done in the Department of General Surgery, Government Medical College, Thrissur, during a period of 1 year from 1st June 2013 to 31st May 2014, with a mean follow-up of 1 year.

Patients presented to Surgery Department with absolute dysphagia due to Carcinoma oesophagus, offered palliative SEMS after confirming the diagnosis by endoscopy and inoperability by CECT. Consented patients were included in the study.

Oesophageal lesion at $>2 \mathrm{~cm}$ below cricopharyngeal junction. Patients with prior history of treatment with chemotherapy or radiotherapy were excluded from the study.

\section{Patients Demographic Details and Tumour Related Factors Recorded. Dysphagia Graded Using Mellow- Pinkas Score-}

- Gd 0- Normal diet.

- Gd 1-Able to swallow some solid food.

- Gd 2- Able to swallow only semisolid food.

- Gd 3- Able to swallow liquids only.

- Gd 4-Absolute dysphagia.

All the procedures were done under topical anaesthesia using 4\% aerosolized lignocaine and sedation was used in selective patients. Under endoscopic guidance, a guide wire was passed across the stricture. If guide wire could not be negotiated, the procedure was abandoned, and the patient offered other forms of palliation. After putting the guide wire if needed, controlled dilatation of stricture was done. Technical success was considered when the stent could be placed across the stricture and there is improvement of dysphagia achieved at least by one grade. Patients were followed up first in 2 weeks after stenting and thereafter monthly either through telephone or as outpatient basis. The mean follow-up period was one year.

\section{Statistical Analysis}

Non-numeric data was expressed in terms of frequencies and numeric data in mean +/_ SD. In order to compare the dysphagia grade before and after stenting, a non-parametric Wilcoxon Signed Rank Test has been carried out. The frequencies have been compared by conducting parametric Chi-square test for goodness of the test. Survival was assessed with Kaplan-Meier curve. Statistical analysis was done on Epi-Info Software.

\section{RESULTS}

34 patients were selected for stenting based on inclusion and exclusion criteria. We were able to place SEMS successfully in
29 patients. The technical success rate was $85.29 \%$. Reasons for failure in 4 patients were due to tight stricture and 1 patient could not withstand the procedure due to poor cardiac performance. In final analysis, there were 29 patients. Mean age of study was 67.5 with a minimum as of 41 and maximum age of 87. Of 29 patients undergone stenting with SEMS, 23 were males (79.3\%) and 6 were females (20.7\%).

Other basic parameters are in the table.(1) $62.1 \%$ of our patients had squamous cell carcinoma and the rest (37.9\%) had adenocarcinoma. Location of the tumour was in middle third of esophagus in 15 (51.7\%), lower third in 9 (31\%) and upper third in 5 (17.5\%). 18 of our patients had metastatic disease and the rest had inoperable locally advanced malignancy. Following the procedure all patients were advised palliative chemotherapy, but majority of our patients refused further treatment. Only 10 individuals received post procedure chemotherapy.

\section{Relief of Dysphagia}

After stenting, all the patients experienced a significant improvement in dysphagia except one. He had oesophageal perforation during the procedure and expired on the 5th day due to sepsis. Clinical success rate was $96.55 \%$. All the patients showed at least one grade improvement in their dysphagia score. Post stent score was Gd 3 in 19 (65.5\%), Gd 2 in 7 (24.1\%), Gd 1 in 2 (6.9\%) and Gd 4 in 1 (3.4\%). Wilcoxon Signed Rank Test ' $z$ ' value was 4.813 (p value $<0.001)$. Median survival was 20 weeks $(95 \%$, CI 12.08 27.91).

\section{Complications (Table 2)}

There was one procedure related mortality due to oesophageal perforation. Incidence of recurrent dysphagia in our study was $17.24 \%$. It was due to stent migration in $2(06.89 \%)$ and tumour overgrowth in 3 (10.34\%). Stent migrations were partial in both patients and managed by inserting a second stent. Bleeding occurred in 4 patients, which were mild in nature and managed by conservative measures. Most common complications following stenting was chest pain. None were severe in nature and was managed by conventional analgesics. 8 patients had reflux symptoms, all of them were stented for lower oesophagus lesions. Mean follow-up period was 1 year and Meier-Kaplan median survival was 20 weeks (12.1 - 27.9 weeks, CI 95\%) Fig. 1.

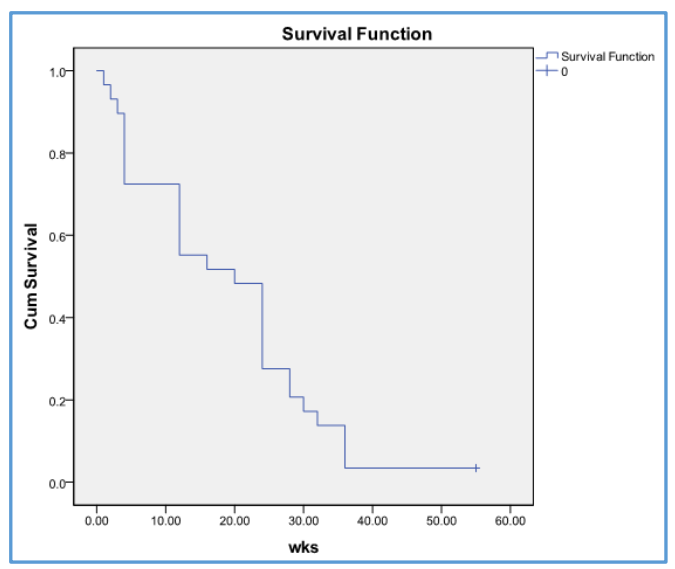

Figure 1. Post Stent Patient Survival in Weeks (Medium Survival 20 Weeks) 


\begin{tabular}{|c|c|}
\hline Patient (n) & 29 \\
Age-Mean & 67.5 \\
Male & $23(79.3 \%)$ \\
Female & $6(20.7 \%)$ \\
& \\
Tumour Histology & $18(62.1 \%)$ \\
Squamous cell carcinoma & $11(37.9 \%)$ \\
Adenocarcinoma & \\
Site & $05(17.5 \%)$ \\
Upper 3 ${ }^{\text {rd }}$ & $15(51.7 \%)$ \\
Middle 3rd & $09(31 \%)$ \\
Lower 3rd & \\
\hline \multicolumn{2}{r|}{ Characteristics } \\
\hline Table 1. Patient Demographics and Tumour \\
\hline Perforation \\
Bleeding & $01(03.4)$ \\
Chest pain & $04(13.7)$ \\
Reflux & $17(58.6)$ \\
Mortality & $08(30.7)$ \\
Tumour overgrowth & $01(03.4)$ \\
Stent migration & $03(10.3)$ \\
\hline \multicolumn{2}{|c|}{ Table 2. Complications- $\boldsymbol{n}(\%)$} \\
\hline
\end{tabular}

\section{DISCUSSION}

Globally, overall 5-year survival of Ca oesophagus is dismal $<10 \%$.(1) In developed nations, it is better and reaches up to $30 \%$ for chemotherapy and radiotherapy and less than $40 \%$ for surgery. $(4,6,7)$ Of this, outlook of locally advanced disease is disappointing with median survival of $5-19$ months and in metastatic disease it is further worse. Our study explores the palliation of dysphagia and survival in a cohort of inoperable Ca oesophagus with absolute dysphagia by SEMS placement. We had a technical success rate of $85.25 \%$ and clinical success rate of $96.55 \%$.

The technical success of oesophageal stenting approaches $100 \%$ and a symptomatic relief is about $82 \%$ to $100 \%$ in various studies. SEMS is a well-accepted palliative procedure for relieving dysphagia.(8) It produces excellent improvement in dysphagia score with minimal complications. A large study from Kenya reported an improvement from a mean present score of 3.3 to a mean post stent score of 1 - 1.8.(9) Our study specifically examines the effect of stenting in absolute dysphagia and we observed an improvement from a median present dysphagia score of 4 and 5 to a mean score of $2.65+/-$ 0.66 , which was statistically significant.

It is a long held belief that SEMS did not affect survival. A non-randomised study comparing radiotherapy and chemotherapy versus SEMS alone, the median survival of SEMS group alone was considerably less (11 months vs. 4 months).(10) But patients recruited for SEMS in these trials were more frail and unfit patients for radio and chemotherapy. Moreover majority of these patients were failed treatment with radio and chemotherapy.

Even though stents did not have cytocidal activity, it may indirectly lengthen the survival by improving nutrition and preventing aspiration. A large cohort study from Kenya reports a median survival of 250 days following SEMS alone for palliation of inoperable carcinoma oesophagus.(9) Median survival data from present study is 20 weeks. The reason for this may be that all of our patients had absolute dysphagia at presentation, which leads to poor general health and nutrition.

This survival data was comparable to other modalities like Photodynamic therapy, Laser therapy and single dose brachytherapy. $(11,12,13)$ Of this, single dose brachytherapy has fewer complications and better quality of life. But SEMS is a better option in patients with limited life expectancy, since it gives excellent immediate palliation of dysphagia.(13)

Even though SEMS is an effective method to relieve dysphagia, it is not without complications. Stent migration was a major complication associated with SEMS with an incidence ranging from 7 to $75 \%$.(14) The risk factors were use of covered stents and stenting for lower oesophageal lesions.(15) Management depended on extent of displacement. Partial displacement was usually managed by inserting an uncovered stent. Complete displacement into stomach necessitated endoscopic removal or through gastrostomy removal.(16)

Other major complications were stent obstruction due to tumour ingrowth or overgrowth, perforation and bleeding. Stent obstruction due to tumour in growth is rare now a days due to widespread use of fully covered SEMS.(17) Tumour overgrowth is relatively common with an incidence ranging between $5 \%$ to $10 \%$. $^{(18)}$

In our study, the overall incidence of recurrent dysphagia was $17 \%$. Tumour ingrowth or overgrowth were the main reasons. Stent migration occurred in 2/29 (6.89) and stent migrations occurred in one patient and was managed by reinsertion of another stent and tumour overgrowth was managed by radiotherapy. An Indian study showed similar observation with $6 \%$ incidence of stent migration and $12 \%$ stent obstruction due to tumour overgrowth.(18) The reason for this low incidence in our study may be the very narrow stricture causing absolute dysphagia and minimal use of dilatation causing the stents to be tightly adherent to the tumour. Even though the incidence of oesophageal perforation is rare,(19) we had one incidence of perforation. Other complications like chest pain, foreign body sensation etc. were not serious enough to warrant stent removal or any other interventions. These complications were managed by analgesics and other conservative methods.

Small sample size and not factoring the disease specific quality of life assessment are the major limitations of our study. Significance of this study is that it specifically examines the effectiveness of SEMS in a group of patients with absolute dysphagia as a primary palliative modality. This aspect is important considering the limited radiation facilities and high cost of chemotherapy. This care can be provided to rural population without much specialist facilities. It establishes the feasibility and safety in a rural setup in a developing country.

\section{CONCLUSION}

We had a durable palliation with minimal mortality and morbidity. Our technical success rate is $85.5 \%$ and clinical success rate is $96.55 \%$. Median survival of our patients is 20 weeks, which is comparable to other modalities. In conclusion, SEMS is an effective method for palliation of grade 4 dysphagia due to carcinoma oesophagus. 


\section{REFERENCES}

[1] Ferlay J, Soerjomataram I, Ervik M, et al. GLOBOCAN 2012 v1.0, Cancer incidence and mortality worldwide: International Agency for Research on Cancer, IARC CancerBase No. 11. Lyon, France: 2013. http://www.globocan.iarc.fr

[2] Samarasam I. Esophageal cancer in India: current status and future perspectives. Int J Adv Med Health Res 2017;4(1):5-10.

[3] Javle M, Ailawadhi S, Yang GY, et al. Palliation of malignant dysphagia in esophageal cancer: a literature-based review. J Support Oncol 2006;4(8):365-73, 379.

[4] Cooper JS, Guo MD, Herskovic A, et al. Chemoradiotherapy of locally advanced esophageal cancer: long-term follow-up of a prospective randomized trial (RTOG 85-01). Radiation Therapy Oncology Group. JAMA 1999;281(17):1623-7.

[5] Baron TH. Expandable metal stents for the treatment of cancerous obstruction of the gastrointestinal tract. N Engl J Med 2001;344(22):1681-7.

[6] Schwer AL, Ballonoff A, McCammon R, et al. Survival effect of neoadjuvant radiotherapy before esophagectomy for patients with esophageal cancer: a surveillance, epidemiology and end-results study. Int J Radiat Oncol Biol Phys 2009;73(2):449-55.

[7] Bedenne L, Michel P, Bouche 0, et al. Chemoradiation followed by surgery compared with chemoradiation alone in squamous cancer of the esophagus: FFCD 9102. J Clin Oncol 2007;25(10):1160-8.

[8] Sarper A, Oz N, Cihangir C, et al. The efficacy of selfexpanding metal stents for palliation of malignant esophageal strictures and fistulas. Eur J Cardiothorac Surg 2003;23(5):794-8.

[9] White RE, Parker RK, Fitzwater JW, et al. Stents as sole therapy for oesophageal cancer: a prospective analysis of outcomes after placement. The Lancet Oncology 2009;10(3):240-6.
[10] Wong SKH, Chiu PWY, Leung SF, et al. Concurrent chemoradiotherapy or endoscopic stenting for advanced squamous cell carcinoma of esophagus: a case control study. Ann Surg Oncol 2008;15(2):57682.

[11] Litle VR, Luketich JD, Christie NA, et al. Photodynamic therapy as palliation for esophageal cancer: experience in 215 patients. Ann Thorac Surg 2003;76(5):1687-93.

[12] Dallal HJ, Smith GD, Grieve DC, et al. A randomized trial of thermal ablative therapy versus expandable metal stents in the palliative treatment of patients with esophageal carcinoma. Gastrointest Endosc 2001;54(5):549-57.

[13] Homs MY, Steyerberg EW, Eijkenboom WM, et al. Single-dose brachytherapy versus metal stent placement for the palliation of dysphagia from oesophageal cancer: multicentre randomised trial. Lancet 2004;364(9444):1497-504.

[14] Sharma P, Kozarek R, Practice Parameters Committee of American College of Gastroenterology. Role of esophageal stents in benign and malignant diseases. Am J Gastroenterol 2010;105(2):258-74.

[15] Warren WH. Palliation of dysphagia. Chest Surg Clin N Am 2000;10(3):605-23.

[16] McManus K, Khan I, McGuigan J. Self-expanding oesophageal stents: strategies for re-intervention. Endoscopy 2001;33(7):601-4.

[17] Na HK, Song HY, Kim JH, et al. How to design the optimal self-expandable oesophageal metallic stents: years of experience in 645 patients with malignant strictures. Eur Radiol 2013;23(3):786-96.

[18] Madhusudhan C, Saluja SS, Pal S, et al. Palliative stenting for relief of dysphagia in patients with inoperable esophageal cancer: impact on quality of life. Diseases of the Esophagus 2009;22(4):331-6.

[19] Xinopoulos D, Dimitroulopoulos D, Moschandrea I, et al. Natural course of inoperable esophageal cancer treated with metallic expandable stents: quality of life and cost-effectiveness analysis. J Gastroenterol Hepatol 2004;19(12):1397-402. 